DESY-97-118

DO-TH 97/14

June 1997

\title{
The Bethe-Heitler Process in Polarized Photon-Nucleon Interactions
}

\author{
T. Gehrmann \\ DESY, Theory Group, \\ D-22603 Hamburg, Germany \\ M. Stratmann \\ Universität Dortmund, Institut für Physik, \\ D-44221 Dortmund, Germany
}

\begin{abstract}
We calculate the Bethe-Heitler cross section for the production of lepton pairs in the field of a longitudinally polarized nucleon, taking into account the lepton masses and the target mass. This process is a dominant background to the detection of open charm from semi-leptonic decay modes, which is a potential probe of the polarized gluon distribution in the nucleon.
\end{abstract}


The knowledge on the spin structure of the nucleon has improved considerably over the past few years. More precise measurements of the spin asymmetry $A_{1}\left(x, Q^{2}\right) \simeq$ $g_{1}\left(x, Q^{2}\right) / F_{1}\left(x, Q^{2}\right)$ in longitudinally polarized deep-inelastic scattering (DIS) of leptons off proton, deuterium and neutron targets [1] yielded valuable information on spin sum rules and on the polarized valence quark distributions. On the theoretical side, it has become possible to perform a consistent analysis of polarized DIS in next-to-leading order (NLO), since the required spin-dependent two-loop splitting functions have been calculated recently [2]. Nevertheless, all NLO analyses [3, [4] have demonstrated, that the available data sets are still not sufficient for an accurate extraction of the spin-dependent sea quark and gluon densities of the nucleon. This is true in particular for the detailed $x$ shape of the spin-dependent gluon distribution, even though a tendency towards a sizeable

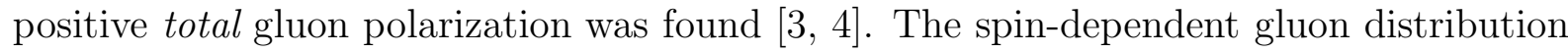
enters the polarized structure functions at leading order (LO) only indirectly via the $Q^{2}$ dependence of $g_{1}$ which could not be studied accurately up to now due to the rather limited kinematical coverage in $\left(x, Q^{2}\right)$ of the present fixed target experiments [1]. Moreover, a direct extraction of the gluon distribution from scaling violations of the polarized structure functions is more involved than in the unpolarized case, as a complicated interplay of quark and gluon contributions to the scaling violations [5] is taking place even at low values of $x$. Clearly, the determination of the polarized gluon distribution is one of the most interesting challenges for future spin physics experiments.

Recently much effort was devoted to examine the feasibility of such measurements at future polarized $p p$ (RHIC, [6]) and $e p$ colliders, one conceivable option for a future HERA upgrade which is currently under discussion [7]. An alternative measurement could be possible at the recently approved COMPASS experiment [8] at CERN or at the proposed E156 experiment [9] at SLAC. The key process studied in these latter fixed target experiments is the production of charmed particles, as the cross section asymmetry for open charm photoproduction $\Delta \sigma^{\gamma N \rightarrow c \bar{c} X} / \sigma^{\gamma N \rightarrow c \bar{c} X}$ provides a clear tool to access the spin- 
dependent gluon distribution due to the dominance of the photon-gluon fusion subprocess $\gamma g \rightarrow c \bar{c}$. Such a measurement at fixed target energies has originally been suggested in the literature in [10] and was further studied in [11]. The charm production induced by partons in the photon (the 'resolved' subprocess, where also the yet experimentally unknown polarized parton distributions of the photon enter) is moreover shown to be negligibly small at the energies available at fixed target experiments $\left(\sqrt{S_{\gamma N}} \lesssim 20 \mathrm{GeV}\right)$ for realistic scenarios of the photonic parton densities [12].

The charmed events can either be detected via their hadronic $D$-meson decays $\left(D^{0} \rightarrow\right.$ $\left.K^{-} \pi^{+}, D^{*+} \rightarrow D^{0} \pi_{\text {soft }}^{+} \rightarrow\left(K^{-} \pi^{+}\right) \pi_{\text {soft }}^{+}, \ldots\right)$, which allow for an efficient background rejection provided a sufficiently good particle identification and energy resolution, or from the observation of decay muons. The hadronic $D$-meson decay channels were used in the recent $\mathrm{H} 1$ and ZEUS measurements [13] and will be employed also in the upcoming COMPASS experiment [8]. The proposed SLAC experiment [9] will use the muonic decay channels, which were first used in the measurements of charm photoproduction by EMC [14.

Obviously, a good understanding of possible background processes yielding charged lepton final states is essential in the latter case. The calculation of asymmetries induced by one of the most important background processes, the photoproduction of charged leptons with circularly polarized photons and longitudinally polarized nucleons via the Bethe-Heitler (BH) mechanism [15] depicted in Fig. 1 is the purpose of this paper. There is, in principle, another source of charged leptons in photon-hadron interactions [16]: the Drell-Yan (DY) process, where the incoming quasi-real photon can either resolve into its hadronic content ('resolved' process) or can act as an elementary particle ('direct' process). For the energies available at the proposed SLAC experiment $\left(E_{\gamma} \lesssim 50 \mathrm{GeV} \Leftrightarrow\right.$ $\left.\sqrt{\mathrm{S}_{\gamma \mathrm{N}}} \lesssim 10 \mathrm{GeV}\right)$, the contribution from the DY mechanism is however expected to be only marginal compared to the $\mathrm{BH}$ process as can be inferred from corresponding analyses in the unpolarized case [16]. Moreover, there is also experimental indication that lepton 

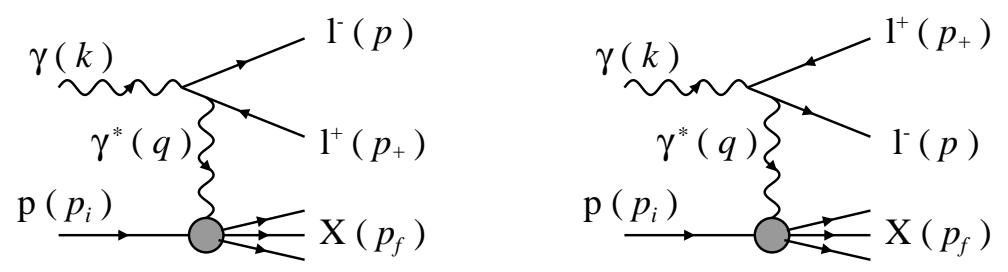

Figure 1: Feynman diagrams for the photoproduction of leptons via the Bethe-Heitler process.

pairs in unpolarized photon-nucleon collisions are produced predominantly by the $\mathrm{BH}$ process. The NA14 experiment, which has studied the photoproduction of $J / \psi$-particles at $E_{\gamma} \approx 90 \mathrm{GeV}$, has found [17] that the lepton pair continuum below the $J / \psi$ resonance is well described by the Bethe-Heitler process only.

Apart from being a background process to the detection of open charm, the polarized $\mathrm{BH}$ cross section is interesting in itself as a probe of the polarized structure functions $g_{1}$ and $g_{2}$. The momenta of the two muons allow for a complete reconstruction of the kinematics and hence a for a measurement in any desired kinematic region (deep inelastic, resonance, elastic). This could be in particular relevant for a measurement of $g_{1}$ in the region of low $x$ and low $Q^{2}$, where the SLAC electroproduction experiments suffered from large pionic backgrounds 18. However, more detailed studies using the formulae derived below will have to be carried out to test the feasibility of such a measurement.

The Bethe-Heitler process in the field of a nucleon can be viewed as the fluctuation of a real photon into an off-shell lepton-antilepton pair, which is put on-shell by interacting with the target nucleon, which is not necessarily left intact (Fig. 1). The interaction with the nucleon is described by the same structure functions appearing in lepton-nucleon scattering. The unpolarized cross section $\mathrm{d} \bar{\sigma}$ for this process was originally calculated long ago by Drell and Walecka in [19] and rederived by Kim and Tsai [20, 21], whose notation we will adopt in the following. Moreover, polarization asymmetries in the Bethe-Heitler 
process with circularly polarized photons onto an unpolarized target due to electroweak interference or due to external fields have been derived in [22].

We consider the production of charged lepton pairs (with lepton mass $m$ ) in the collision of a photon beam off a nucleon target $N$ of mass $m_{i}$ and spin direction $S^{\alpha}$ :

$$
\gamma(k)+N\left(p_{i}\right) \longrightarrow l^{+}\left(p_{+}\right)+l^{-}(p)+X\left(p_{f}\right)
$$

The hadronic final state $X$ has an invariant mass $m_{f}$ and the four-momentum transfer to the target is denoted by $q^{\alpha}=p_{f}^{\alpha}-p_{i}^{\alpha}=k^{\alpha}-p^{\alpha}-p_{+}^{\alpha}$ with $-q^{2} \equiv Q^{2}>0$.

The hadronic tensor is defined in terms of two spin-independent structure functions $W_{1,2}$ and two spin-dependent ones, $G_{1,2}$, appearing in the symmetric and anti-symmetric part of $W^{\mu \nu}$, respectively:

$$
\begin{aligned}
W^{\mu \nu}= & \frac{1}{4 \pi m_{i} e^{2}} \sum_{f}\left\langle p_{i}, S\left|J^{* \mu}(0)\right| f\right\rangle\left\langle f\left|J^{\nu}(0)\right| p_{i}, S\right\rangle(2 \pi)^{4} \delta^{4}\left(q+p_{i}-p_{f}\right) \\
= & -\left(g^{\mu \nu}-\frac{q^{\mu} q^{\nu}}{q^{2}}\right) W_{1}\left(\nu, q^{2}\right)+\frac{1}{m_{i}^{2}}\left(p_{i}^{\mu}-\frac{p_{i} \cdot q}{q^{2}} q^{\mu}\right)\left(p_{i}^{\nu}-\frac{p_{i} \cdot q}{q^{2}} q^{\nu}\right) W_{2}\left(\nu, q^{2}\right) \\
& +\frac{i}{m_{i}^{2}} \epsilon^{\mu \nu \rho \sigma} q_{\rho}\left(S_{\sigma}\left(G_{1}\left(\nu, q^{2}\right)+\frac{p_{i} \cdot q}{m_{i}^{2}} G_{2}\left(\nu, q^{2}\right)\right)-\frac{S \cdot q}{m_{i}^{2}} p_{i \sigma} G_{2}\left(\nu, q^{2}\right)\right)
\end{aligned}
$$

where $\nu=p_{i} \cdot q / m_{i}$.

We define the longitudinally polarized and unpolarized cross sections in the usual way via

$$
\mathrm{d} \Delta \sigma \equiv \frac{1}{2}\left(\mathrm{~d} \sigma^{\vec{\gtrless}}-\mathrm{d} \sigma^{\rightrightarrows}\right), \quad \mathrm{d} \bar{\sigma} \equiv \frac{1}{2}\left(\mathrm{~d} \sigma^{\rightleftarrows}+\mathrm{d} \sigma^{\rightrightarrows}\right)
$$

(the arrows denote the spin directions of beam and target) such that the measurable cross section asymmetry becomes

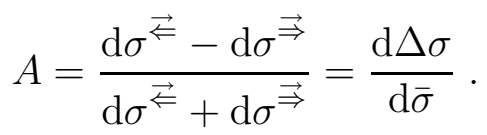

We present our results in the experimentally relevant target rest frame and denote the energy of the photon in this frame by $K$ and the energies of the lepton and antilepton by 
$E$ and $E_{+}$, respectively. The polarized $\mathrm{BH}$ cross section finally reads

$$
\mathrm{d} \Delta \sigma=e^{6} \frac{m_{i}}{4\left(k \cdot p_{i}\right)} \frac{\mathrm{d}^{3} p}{E} \frac{\mathrm{d}^{3} p_{+}}{E_{+}} \frac{1}{2^{6} \pi^{5}} \frac{1}{q^{4}} L_{\mu \nu}^{A} W_{A}^{\mu \nu},
$$

where the contraction of the antisymmetric parts of the leptonic and hadronic tensors in (回) is given by

$$
\begin{aligned}
&-\frac{m_{i}^{2}}{4} L_{\mu \nu}^{A} W_{A}^{\mu \nu}= G_{1}\left(q^{2}, m_{f}^{2}\right)\left[\frac{H_{1}}{\left(p_{+} \cdot k\right)^{2}}+\frac{B_{1}}{\left(p_{+} \cdot k\right)}+C_{1}+D_{1}\left(p_{+} \cdot k\right)+E_{1}\left(p_{+} \cdot k\right)^{2}\right] \\
&+\frac{q^{2}}{m_{i}} G_{2}\left(q^{2}, m_{f}^{2}\right)\left[\frac{H_{2}}{\left(p_{+} \cdot k\right)^{2}}+\frac{B_{2}}{\left(p_{+} \cdot k\right)}+C_{2}+D_{2}\left(p_{+} \cdot k\right)\right]
\end{aligned}
$$

with

$$
\begin{aligned}
H_{1}= & -m^{2}\left[K q^{2}+(k \cdot p) \Delta+\frac{(k \cdot p)^{2}}{K}-\frac{(k \cdot p) q^{2}}{2 m_{i}}\right] \\
B_{1}= & \frac{(k \cdot p)^{2}}{K}+\left[\frac{1}{K}\left(q^{2}-2 m^{2}\right)-\frac{q^{2}}{2 m_{i}}+\Delta\right](k \cdot p)+\frac{K q^{4}}{2(k \cdot p)} \\
& +q^{2}\left[\frac{m^{2}}{2 m_{i}}-(E-K)\right]-m^{2} \Delta \\
C_{1}= & \frac{1}{K}\left[(k \cdot p)-2 m^{2}\right]+\frac{1}{(k \cdot p)}\left[\left(q^{2}-m^{2}\right)\left(\Delta-\frac{q^{2}}{2 m_{i}}\right)+E q^{2}\right]-\frac{1}{(k \cdot p)^{2}} K q^{2} m^{2} \\
D_{1}= & \frac{1}{K}+\frac{1}{(k \cdot p)}\left[\frac{1}{K}\left(q^{2}-2 m^{2}\right)+\Delta-\frac{q^{2}}{2 m_{i}}\right]+\frac{m^{2}}{(k \cdot p)^{2}}\left[\frac{q^{2}}{2 m_{i}}-\Delta\right] \\
E_{1}= & \frac{1}{K(k \cdot p)}\left[1-\frac{m^{2}}{(k \cdot p)}\right] \\
H_{2}= & m^{2}(k \cdot p) \\
B_{2}= & \frac{(k \cdot p)}{K}\left[E-K+\Delta-\frac{q^{2}}{2 m_{i}}\right]+m^{2}-\frac{q^{2}}{2} \\
C_{2}= & \frac{q^{2}}{2 m_{i} K}-\frac{\Delta}{K}-1+\frac{1}{(k \cdot p)}\left[m^{2}-\frac{q^{2}}{2}\right] \\
D_{2}= & \frac{1}{(k \cdot p)}\left[\frac{m^{2}}{(k \cdot p)}-\frac{E}{K}\right]
\end{aligned}
$$

where we have introduced $\Delta \equiv\left(m_{f}^{2}-m_{i}^{2}\right) /\left(2 m_{i}\right)$.

Finally, we calculate the cross section where only one of the leptons, say the $l^{-}(p)$, is observed by integrating over $d^{3} p_{+}$in Eq. (田). This integration is most conveniently 
performed in the frame where $\vec{k}-\vec{p}$ is at rest and both the vectors $\vec{k}$ and $\vec{p}$ lie in the $x z$ plane [21]. Vectors, momenta and energies in this special frame are denoted by a subscript $s$. The angle between $\vec{k}_{s}$ and the $z$ axis is denoted by $\Theta_{k}$, the angle between $\vec{p}_{+s}$ and the $z$ axis by $\Theta_{+}$. The projection of $\vec{p}_{+s}$ onto the $x y$ plane and the $x$ axis forms the angle $\phi$. The chosen frame has the advantage that the integration over $\phi$ can be straightforwardly carried out analytically, following closely the unpolarized calculation of Kim and Tsai [20, 21], where more details can be found.

By defining an auxiliary vector

$$
U^{\alpha} \equiv p_{+}^{\alpha}+p_{f}^{\alpha}=k^{\alpha}+p_{i}^{\alpha}-p^{\alpha}
$$

with

$$
U=\sqrt{U^{2}}=\sqrt{m^{2}+m_{i}^{2}+2 m_{i}(K-E)-2(k \cdot p)}
$$

in the target rest frame, all particle energies, momenta and angles in the special frame can be expressed in terms of observables as follows:

$$
\begin{aligned}
K_{s} & =\frac{K m_{i}-(k \cdot p)}{U} \\
E_{+s} & =\frac{U^{2}+m^{2}-m_{f}^{2}}{2 U} \\
p_{+s} & =\sqrt{E_{+s}^{2}-m^{2}} \\
p_{i s} & =\frac{m_{i}}{U} \sqrt{K^{2}+E^{2}-m^{2}+2[(k \cdot p)-K E]} \\
E_{s} & =\frac{(k \cdot p)+m_{i} E-m^{2}}{U} \\
\cos \Theta_{k} & =\frac{K_{s}-E_{s}}{p_{i s}}+\frac{(k \cdot p)}{K_{s} p_{i s}} \\
\cos \Theta_{+} & =\frac{1}{p_{+s} p_{i s}}\left[\frac{q^{2}}{2}-m^{2}+(k \cdot p)+E_{+s}\left(K_{s}-E_{s}\right)\right] .
\end{aligned}
$$

The cross section (4) for detecting only the lepton $l^{-}(p)$ finally reads $\left(t=-q^{2}\right)$ :

$$
\frac{\mathrm{d} \Delta \sigma}{\mathrm{d} \Omega \mathrm{d} p}=-\frac{\alpha^{3}}{2 \pi m_{i}^{2}} \int_{m_{i}^{2}}^{(U-m)^{2}} \mathrm{~d} m_{f}^{2} \int_{t_{\min }}^{t_{\max }} \mathrm{d} t \frac{p^{2}}{K E U q^{4} p_{i s}}
$$




$$
\begin{aligned}
\times & G_{1}\left(q^{2}, m_{f}^{2}\right)\left\{H_{1} \frac{W}{Y^{3} K_{s}^{2}}+B_{1} \frac{1}{Y K_{s}}+C_{1}+D_{1} K_{s} W+E_{1} K_{s}^{2} \frac{3 W^{2}-Y^{2}}{2}\right\} \\
& \left.+\frac{q^{2}}{m_{i}} G_{2}\left(q^{2}, m_{f}^{2}\right)\left\{H_{2} \frac{W}{Y^{3} K_{s}^{2}}+B_{2} \frac{1}{Y K_{s}}+C_{2}+D_{2} K_{s} W\right\}\right],
\end{aligned}
$$

where we have introduced

$$
\begin{aligned}
W & =E_{+s}-p_{+s} \cos \Theta_{+} \cos \Theta_{k} \\
Y & =\sqrt{m^{2} \sin ^{2} \Theta_{k}+\left(p_{+s} \cos \Theta_{+}-E_{+s} \cos \Theta_{k}\right)^{2}} .
\end{aligned}
$$

The integration limits $t_{\min , \max }$ in (অ) are given by

$$
t_{\max }^{\max }=-2 m^{2}+2(k \cdot p)+2 E_{+s}\left(K_{s}-E_{s}\right) \pm 2 p_{+s} p_{i s}
$$

For completeness, it should be mentioned that the deeply inelastic limit of the formfactors $G_{1}$ and $G_{2}$ appearing in Eqs. (四), (畐), and (7) is related to the commonly used polarized structure functions $g_{1}$ and $g_{2}$ by

$$
\frac{\nu}{m_{i}} G_{1}\left(\nu, q^{2}\right)=g_{1}\left(x, Q^{2}\right), \quad\left(\frac{\nu}{m_{i}}\right)^{2} G_{2}\left(\nu, q^{2}\right)=g_{2}\left(x, Q^{2}\right) .
$$

Finally, the elastic contribution to these form factors, relevant in particular for the calculation of the single lepton inclusive cross section (7) can be expressed as [23]

$$
\begin{aligned}
G_{1}^{\mathrm{el}}\left(\nu, q^{2}\right) & =\frac{G_{M}\left(q^{2}\right)}{2(1+\tau)}\left[G_{E}\left(q^{2}\right)+\tau G_{M}\left(q^{2}\right)\right] m_{i} \delta\left(\nu+\frac{q^{2}}{2 m_{i}}\right), \\
G_{2}^{\mathrm{el}}\left(\nu, q^{2}\right) & =\frac{G_{M}\left(q^{2}\right)}{4(1+\tau)}\left[G_{E}\left(q^{2}\right)-G_{M}\left(q^{2}\right)\right] m_{i} \delta\left(\nu+\frac{q^{2}}{2 m_{i}}\right),
\end{aligned}
$$

with $\tau=-q^{2} /\left(4 m_{i}^{2}\right)$ and $G_{E, M}$ being the elastic nucleon form factors. Using the dipole parameterization [21]

$$
G_{E}\left(q^{2}\right)=\frac{1}{\mu} G_{M}\left(q^{2}\right)=\left(1-q^{2} /\left(0.71 \mathrm{GeV}^{2}\right)\right)^{-2},
$$

with $\mu$ representing the magnetic moment of the nucleon, these form factors yield good agreement with the experimental data on longitudinally polarized elastic electron-proton scattering [24]. 
The formulae (四) and (17) derived above enable, in combination with Eqs.(2.1) and (2.7) of [21], a complete calculation of the Bethe-Heitler cross section with two (one) observed leptons in polarized photon-nucleon collisions, including all effects of lepton and target masses. They are in a form similar to the unpolarized cross sections [20, 21] and can be readily implemented into Monte Carlo simulations of lepton production in photon-nucleon collisions, relevant for realistic background estimates to the detection of open charm - and hence to a measurement of the polarized gluon distribution [9].

Moreover, the Bethe-Heitler cross section with two detected leptons can in principle be used for a measurement of the polarized structure functions $g_{1}$ and $g_{2}$ in any desired kinematics. The feasibility of such a measurement is still to be demonstrated, and the formulae derived in this paper can be used to test the sensitivity of the polarized BetheHeitler process on the polarized structure functions for realistic experimental kinematics.

In summary, we have presented a complete calculation of the Bethe-Heitler photoproduction of lepton pairs in the field of a longitudinally polarized nucleon and given analytic expressions, including all lepton and target mass terms, for the pair production and the single lepton inclusive cross sections.

\section{Acknowledgements}

We are grateful to P. Bosted for drawing our attention to the relevance of the polarized Bethe-Heitler process and for numerous helpful discussions. The work of M.S. has been supported in part by the 'Bundesministerium für Bildung, Wissenschaft, Forschung und Technologie', Bonn.

\section{References}


[1] A recent overview of the experimental status can be found for example in G. Mallot, proceedings of the "12th International Symposium on High Energy Spin Physics (SPIN '96)", Amsterdam 1996, eds. C.W. de Jager et al., World Scientific (Singapore, 1997), p.44.

[2] R. Mertig and W.L. van Neerven, Z. Phys. C70, 637 (1996);

W. Vogelsang, Phys. Rev. D54, 2023 (1996); Nucl. Phys. B475, 47 (1996).

[3] M. Glück, E. Reya, M. Stratmann, and W. Vogelsang, Phys. Rev. D53, 4775 (1996);

T. Gehrmann and W.J. Stirling, Phys. Rev. D53, 6100 (1996);

G. Altarelli, R.D. Ball, S. Forte, and G. Ridolfi, Nucl. Phys. B496, 337 (1997).

[4] SMC Collaboration, D. Adams et al., preprint CERN-PPE-97-022 (hep-ex/9702005), submitted to Phys. Rev. D;

SLAC-E154 Collaboration, K. Abe et al., preprint SLAC-PUB-7461 hepph/9705344), to appear in Phys. Lett. B.

[5] T. Gehrmann and W.J. Stirling, Phys. Lett. B365, 347 (1996).

[6] RHIC-SPIN Collaboration, M. Beddo et al., proposal, BNL, 1992.

[7] J. Feltesse and A. Schäfer, Proceedings of the workshop "Future Physics at HERA", Hamburg 1995/96, eds. G. Ingelman, A. De Roeck and R. Klanner, DESY (Hamburg, 1996), p.757ff.

[8] COMPASS Collaboration, G. Baum et al., proposal, CERN/SPSLC 96-14.

[9] SLAC-E156 Collaboration, R.G. Arnold et al., proposal, SLAC, 1997.

[10] M. Glück and E. Reya, Z. Phys. C39, 569 (1988).

[11] G. Altarelli and W.J. Stirling, Particle World 1, 40 (1989);

M. Glück, E. Reya, and W. Vogelsang, Nucl. Phys. B351, 579 (1991); 
S.I. Alekhin, V.I. Borodulin, and S.F. Sultanov, Int. J. Mod. Phys. A8, 1603 (1993);

S. Keller and J.F. Owens, Phys. Rev. D49, 1199 (1994).

[12] M. Stratmann and W. Vogelsang, Z. Phys. C74, 641 (1997).

[13] H1 Collaboration, C. Adloff et al., Z. Phys. C72, 593 (1996);

ZEUS Collaboration, J. Breitweg et al., Phys. Lett. B401, 192 (1997).

[14] EMC Collaboration, J.J. Aubert et al., Nucl. Phys. B213, 31 (1983).

[15] H.A. Bethe and W. Heitler, Proc. R. Soc. A146, 83 (1934).

[16] R.L. Jaffe, Phys. Rev. D4, 1507 (1971);

A. Vourdas, J. Phys. G6, 789 (1980);

J. Busenitz and J.D. Sullivan, Phys. Rev. D24, 1794 (1981).

[17] NA14 Collaboration, R. Barate et al., Z. Phys. C33, 505 (1987).

[18] P. Bosted, private communication.

[19] S.D. Drell and J.D. Walecka, Ann. Phys. (NY) 28, 18 (1964).

[20] K. Kim and Y.-S. Tsai, Phys. Lett. 40B, 665 (1972).

[21] Y.-S. Tsai, Rev. Mod. Phys. 46, 815 (1974), Erratum 49, 421 (1977).

[22] V.M. Kuznetsov and A.P. Potylitsyn, Sov. J. Nucl. Phys. 27, 79 (1978);

H. Konashi, K. Ushio, and Y. Yokoo, Prog. Theor. Phys. 62, 1062 (1979).

[23] A.I. Akhiezer et al., Sov. Phys. JETP 6, 588 (1958);

N. Dombey, Rev. Mod. Phys. 41, 236 (1969);

T.V. Kuchto and N.M. Shumeiko, Nucl. Phys. B219, 412 (1983).

[24] M.J. Alguard et al., Phys. Rev. Lett. 37, 1258 (1976). 